\title{
Effect of stromal cell-derived factor-1 on myocardial apoptosis and cardiac function recovery in rats with acute myocardial infarction
}

\author{
YUANYUAN LIU, SONGTAO GAO, ZHENG WANG, YAN YANG, HONG HUO and XUEFENG TIAN \\ Department of Cardiology, Heilongjiang Provincial Hospital, Harbin, Heilongjiang 150036, P.R. China
}

Received March 9, 2016; Accepted August 25, 2016

DOI: $10.3892 /$ etm. 2016.3770

\begin{abstract}
The aim of the study was to investigate the effect of stromal cell-derived factor-1 (SDF-1) on myocardial apoptosis and cardiac function recovery in rats with acute myocardial infarction (AMI) and the mechanism of the Toll-like receptor (TLR)-4/nuclear factor- $\kappa \mathrm{B}(\mathrm{NF}-\kappa \mathrm{B})$ signaling pathway. A total of 64 healthy male $\mathrm{F} 344$ rats were randomly divided into the sham operation, model, SDF-1 intervention and SDF-1 antibody groups, with 16 rats in each group. The method of Olivette was used to establish the AMI model by ligation of the left anterior descending artery. Day 1 after establishing the animal model, the rats in the SDF-1 intervention group were injected with $10 \mu \mathrm{l}$ recombinant SDF-1 (400 ng/ml) in five regions including the myocardial infarction area and the four surrounding areas. The rats in the model group were injected with $10 \mu 1$ normal saline including the myocardial infarction area and the four surrounding areas, and those in the SDF-1 antibody group were injected with $1 \mathrm{ml}$ SDF-1 antibody $(2 \mu \mathrm{g} / \mathrm{ml})$. Four rats were sacrificed after 1, 3, 7 and 14 days after the intervention, and the analysis was carried out. TUNEL in situ labeled apoptotic cells were used for cell counting, and immunohistochemical staining was performed to measure vascular density. The animal echocardiographic measurement was for the left ventricular end-diastolic diameter (LVEDd), left ventricular end-systolic diameter (LVESd), left ventricular fractional shortening (FS) and ejection fraction (EF) values. The results showed that the number of apoptotic cells in the SDF-1 treatment group was significantly lower than those in the other groups at each time-point. The vessel densities in the 3-14 days were significantly greater than those in other groups. At each time-point, the LVEDd and LVESd values were smaller compared with the model group, but greater than the sham operation group and decreased over time. FS and EF values were higher than those in the model
\end{abstract}

Correspondence to: Dr Xuefeng Tian, Department of Cardiology, Heilongjiang Provincial Hospital, 82 Zhongshan Road, Harbin, Heilongjiang 150036, P.R. China

E-mail: tian_xuefeng1@163.com

Key words: stromal cell-derived factor-1, acute myocardial infarction, myocardial apoptosis, cardiac function, Toll-like receptor group at each time-point, but less than those of the sham operation group and increased over time. The expression levels of TLR-4 and NF- $\kappa \mathrm{B}$ at each time-point were significantly higher than those of the remaining groups $(\mathrm{p}<0.05)$. In conclusion, SDF-1 is capable of decreasing the apoptosis of cardiac muscle cells in AMI, promoting angiogenesis and improving cardiac function, which may be associated with the activation of the TLR-4/NF- $\mathrm{B}$ signaling pathway.

\section{Introduction}

Acute myocardial infarction (AMI) stem cell transplantation as a type of treatment is on the increase. A number of studies such as the REPAIR-AMI, REGENT and BOOST indicated that stem cell therapy improved the ischemic myocardial remodeling outcomes. Benefit was also associated with the degree of ischemia (1-3).

For myocardial regeneration, the bone marrow mesenchymal stem cells or endothelial progenitor cells (EPCs) need to be homed to the infarcted area and directional differentiation into cardiomyocytes, in order to have normal physiological function and therapeutic effect. Various differentiation inducing factors such as stromal cell-derived factor-1 (SDF-1), insulin like growth factor-1 and granulocyte colony-stimulating factor, play a key role in stem cell directional migration and differentiation $(4,5)$. However, previous studies focused on the differentiation-inducing factor promoting the stem cell therapy effect $(6,7)$.

The aim of the present study was to examine whether SDF-1 affects the apoptosis of AMI, angiogenesis and heart function changes and the relevant function mechanisms involved.

\section{Materials and methods}

Animals. Sixty-four healthy male F344 rats were purchased from Silaike Experimental Animal Co., Ltd. (Shanghai, China), with gestational age of 6-8 months and body weight of $250 \pm 15 \mathrm{~g}$. The rats were kept in cages and given unrestricted access to food and water, with 12-h light/dark cycle, a temperature of $20 \pm 3^{\circ} \mathrm{C}$, and humidity of $53 \pm 5 \%$.

AMI model. According to the Olivette method, the rats were fixed in supine position after being anesthetized with ether and anesthesia was maintained by intraperitoneal 
injection of ketamine $(100 \mathrm{mg} / \mathrm{kg})$. ALC-V8 ventilator (Alcott Biotechnology Co., Ltd., Shanghai, China) was used to support respiration and an ECG instrument was used for ECG monitoring. The rats were operated with chest median longitudinal incision, and tissues were separated by layer separation. Thoracotomy was conducted along the fourth intercostal space, and the pericardium was separated to expose the heart. A needle was inserted in the junction below the edge of the atrioventricular, left atrial appendage and conus and 6-0. Prolene was used for ligature of the left anterior descending branch. The left ventricular anterior wall lost its original luster, and had a pale appearance, with a weak pulse and the ECG showed ST segment and Q wave changes, indicating that AMI models were successfully manufactured. The chest was observed continually for 3-5 min until a stable cycle was obtained. The rats in the sham operation group had only thread in the corresponding position without ligation.

Experimental grouping. The rats were randomly divided into the sham operation, model, SDF-1 intervention and SDF-1 antibody groups, with 16 rats in each group. On day 1 after the model was successfully established, the rats in the SDF-1 intervention group were injected with $10 \mu \mathrm{l}$ recombinant SDF-1 (400 ng/ml; Peprotech, Inc., Rocky Hill, NJ, USA) in five regions including the myocardial infarction area and the four surrounding areas. The rats in the model group were injected with $10 \mu \mathrm{l}$ normal saline including the myocardial infarction area and the four surrounding areas, and those in the SDF-1 antibody group were injected with $1 \mathrm{ml} \mathrm{SDF-1} \mathrm{antibody}$ (2 $\mu \mathrm{g} / \mathrm{ml}$; United States Biological, Swampscott, MA, USA). Four rats were sacrificed (5\% isoflurane followed by cervical dislocation) 1, 3, 7 and 14 days after the intervention, and the analysis was carried out.

Terminal deoxynucleotidyl-transferase-mediated dUTP nick end-labelling (TUNEL). TUNEL method was used to count in situ labeling apoptotic cells. According to the instructions of the kit procedures, the heart was fixed with $4 \%$ polyformaldehyde phosphate solution, dehydrated and paraffin-embedded, and sectioned ( $4 \mu \mathrm{m})$. The interval to the long axis was $100 \mu \mathrm{m}$ perpendicular. After the conventional xylene dewaxing and gradient ethanol hydration, the terminal deoxynucleotidyl transfer enzyme-mediated method was used. TUNEL in situ was used to mark myocardial cell apoptosis. Normal myocardial nuclei were blue, while apoptotic-positive myocardial nuclei were brown. The proportion of the number of apoptotic-positive cardiac muscle cells to the total number of nuclei in 5 random high fields of view (x40) were counted under an optical microscope (Olympus, Tokyo, Japan), and the mean value was obtained.

Immunohistochemical staining. CD34 immunohistochemical staining measurement for microvessel density (MVD) was counted using the Image-Pro Plus 5.0 system (Media Cybernetics, Inc., Rockville, MD, USA) with reference to the method used by Weidner (8). First, optical microscope at a magnification of $x 20$ scanned the entire section, and five regions of highest vascular density as 'hot spots' around the infarction area were selected. The number of vessels was counted with dyed brown inside the hot spots under a magnification of $x 200$ using the optical microscope. The endothelial cells, the cluster of endothelial cells, and cabled endothelial cells were counted as one blood vessel without taking the lumen into account or if there were any red blood cells. The vessel with a luminal diameter of $>20 \mu \mathrm{m}$ or thick muscle layer was not included. Each sample was selected for five views (x200) counting the number of microvessels.

HP SONOS 5500 animal ultrasonic echocardiography (Hewlett-Packard, Palo Alto, CA, USA) was used to measure the left ventricular end-diastolic diameter (LVEDd), left ventricular end-systolic diameter (LVESd), left ventricular fractional shortening (FS) and ejection fraction (EF) value. The probe was $11.5 \mathrm{MHz}$ and placed in the left parasternal intercostal 4 for 5 of the rats. According to the American Heart Association ultrasound recommended guidelines (9), the measurement was completed within at least three consecutive cardiac cycles. The maximum and minimum of ventricular volume for the diastolic and systolic ends were chosen, and the capacity measurements were performed using the modified Simpson's method. Ketamine $(50 \mathrm{mg} / \mathrm{kg}$ ) was used for sedation prior to the examination.

Western blot analysis. The expression level of Toll-like receptor (TLR)-4 and nuclear factor- $\kappa \mathrm{B}(\mathrm{NF}-\kappa \mathrm{B})$ were selected. WIP tissue cell lysis was used for cell protein extraction (Beijing Biosynthesis Biotechnology Co., Ltd., Beijing, China). After measurement of protein concentration using the BCA protein determination kit (Beyotime Institute of Biotechnology, Shanghai, China), $30 \mu \mathrm{g}$ protein sample was added with the sample buffer and boiled for denaturation at $95^{\circ} \mathrm{C}$ for $5 \mathrm{~min}$. Ten percent of SDS-PAGE gel was used for electrophoresis, and the protein was transferred to a PVDF membrane (both from Solarbio, Beijing, China). Then, $5 \%$ milk was used to block in a shaker at $25^{\circ} \mathrm{C}$ for $2 \mathrm{~h}$. Rabbit anti-mouse TLR-4 polyclonal antibody (1:1,000; Affinity Biosciences, Inc., Cincinnati, $\mathrm{OH}$, USA; catalog no. AF7017), rabbit anti-mouse NF-kBp65 polyclonal antibody (1:200; Wuhan Boster Biological Engineering Co., Ltd., Wuhan, China; catalog no. PB0321) and negative control rabbit anti-mouse GAPDH IgG antibody $(1: 1,000$; ZSGB-BIO Technology Co., Ltd., Beijing, China; catalog no. TA309157) were used for incubation at $4^{\circ} \mathrm{C}$ overnight. TBST buffer was used for washing, followed by incubation of the membrane with horseradish peroxidase-labeled goat antirabbit IgG (1:5,000; ZSGB-BIO Technology Co., Ltd.; catalog no. ZDR-5306) at $25^{\circ} \mathrm{C}$ in the shaker for $2 \mathrm{~h}$. After washing the membrane with TBST buffer, Super ECL Plus sensitive luminous liquid was added and placed in the dark (Applygen Technologies, Inc., Beijing, China). After exposure, the image was recorded using the SensiAnsys gel imaging system. With the gray value, the corresponding gray level ratio of target protein bands with reference protein bands was used to calculate each protein relative expression level.

Statistical analysis. SPSS software (Chicago, IL, USA) was used for data analysis and processing. Mean \pm standard deviation was used for quantitative data. Single-factor ANOVA analysis was used for group comparison, and the number of cases or percentage were determined for the qualitative data, and $\chi^{2}$ test was used for comparison between the groups. $\mathrm{P}<0.05$ was considered statistically different. 
Table I. Comparison of the number of apoptotic cell at each time-point $(\%)$.

\begin{tabular}{lrrcc}
\hline Groups & 1 day & 3 days & 7 days & 14 days \\
\hline Sham operation & $0.6 \pm 0.1$ & $0.6 \pm 0.1$ & $0.6 \pm 0.1$ & $0.6 \pm 0.1$ \\
Model & $43.7 \pm 6.6$ & $68.9 \pm 7.4$ & $77.5 \pm 9.2$ & $80.4 \pm 10.3$ \\
SDF-1 intervention & $26.4 \pm 4.2$ & $35.5 \pm 3.7$ & $27.8 \pm 3.5$ & $23.3 \pm 3.2$ \\
SDF-1 antibody & $50.6 \pm 5.7$ & $73.5 \pm 7.3$ & $82.1 \pm 8.8$ & $86.7 \pm 12.4$ \\
F & 6.324 & 6.648 & 7.553 & 7.964 \\
P-value & $<0.001$ & $<0.001$ & $<0.001$ & $<0.001$ \\
\hline
\end{tabular}

SDF-1, stromal cell-derived factor-1.
Table II. Comparison of the microvessel density at different time-points (/HP).

\begin{tabular}{lrrrr}
\hline Groups & 1 day & 3 days & 7 days & 14 days \\
\hline Sham operation & $1.5 \pm 0.3$ & $1.4 \pm 0.4$ & $1.3 \pm 0.2$ & $1.6 \pm 0.5$ \\
Model & $0.9 \pm 0.2$ & $0.8 \pm 0.3$ & $1.2 \pm 0.4$ & $1.0 \pm 0.3$ \\
SDF-1 intervention & $0.8 \pm 0.3$ & $5.5 \pm 1.2$ & $8.6 \pm 1.5$ & $9.3 \pm 2.2$ \\
SDF-1 antibody & $0.4 \pm 0.1$ & $0.3 \pm 0.1$ & $0.5 \pm 0.1$ & $0.6 \pm 0.1$ \\
F & 5.436 & 5.647 & 5.968 & 6.302 \\
P-value & $<0.001$ & $<0.001$ & $<0.001$ & $<0.001$ \\
\hline
\end{tabular}

SDF-1, stromal cell-derived factor-1.
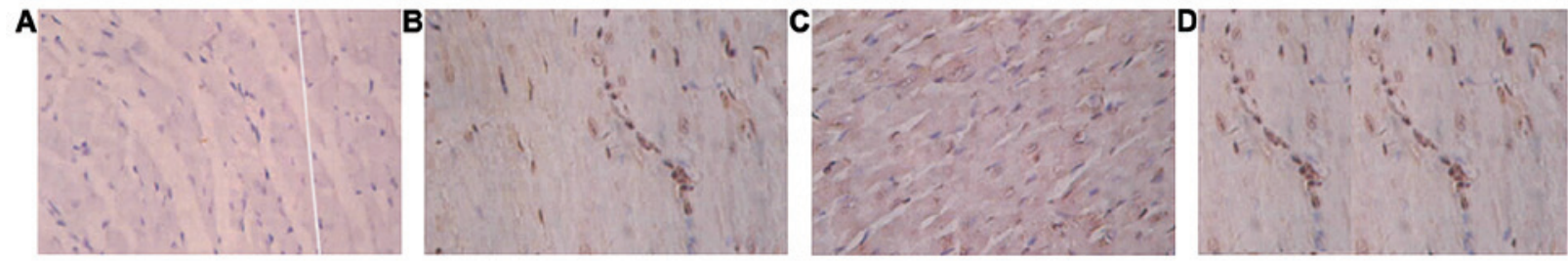

Figure 1. Detection of the number of apoptotic cells in 7 days with TUNEL method. (A) Sham operation group, (B) model group, (C) SDF-1 intervention group and (D) SDF-1 antibody group. TUNEL, terminal deoxynucleotidyl-transferase-mediated dUTP nick end labelling; SDF-1, stromal cell-derived factor-1.

\section{Results}

Comparison of the number of apoptotic cells at each time-point. There was no obvious apoptosis in the sham operation group. The number of apoptotic cells increased with the extension of time in the model group. The number of apoptotic cells of the SDF-1 treatment group was significantly lower at each time-point than the other groups, and the number of apoptotic cells reached their peak on the $3 \mathrm{rd}$ day. The number of apoptotic cells were reduced further after 7 and 14 days. The number of apoptotic cells in the SDF-1 antibody group was significantly higher $(\mathrm{p}<0.05)$ (Table I and Fig. 1).

Comparison of MVD at different time-points. The blood vessel density in the SDF-1 intervention group was significantly higher than that in the remaining groups. The vessel density of the SDF-1 antibody group was significantly smaller at each time-point $(\mathrm{p}<0.05)$, (Table II).

Comparison of cardiac ultrasound parameters. LVEDd and LVESd values of each time-point in the model group were significantly higher than those in the sham operation group, and significantly increased with time. The values of each time-point in the SDF-1 intervention group were smaller compared with those of the model group, but larger than those in the sham operation group and decreased with time. The values in the SDF-1 antibody group were significantly largest in each time-point and increased with time $(\mathrm{p}<0.05)$. The FS and $\mathrm{EF}$ values in model group were significantly smaller than those in the sham-operated group and decreased over time. Those in the SDF-1 treatment group at each time-point were higher than those of the model group, but smaller than those of the sham operation group and increased over time. The
FS and EF values in the anti-SDF-1 antibody group at each time-point were significantly minimum and decreased with time extended ( $\mathrm{p}<0.05)$ (Fig. 2).

Comparison of the expression levels of TLR-4 and NF- $\kappa B$. The expression levels of TLR-4 and NF- $\kappa$ B in the SDF-1 intervention group were significantly higher than those in the other groups at each time-point and increased with time $(\mathrm{p}<0.05)$. In other groups, the differences were not statistically significant ( $\mathrm{p}>0.05$ ), as shown in Table III and Fig. 3 .

\section{Discussion}

SDF-1 is a type of CXC chemokine, which is the only receptor for CXCR4 and is increased significantly in the early stage of myocardial infarction. It can be sustained for 56 days after infarction and is expressed in the hematopoietic stem cell surface (10). Reconstruction of SDF-1 expression in the cell around the infarction area can promote $\mathrm{CD} 117^{+}$and $\mathrm{CD} 34^{+}$ stem cells gradually homing to the myocardial infarction area (11). Nevertheless, only a small fraction of bone marrow mesenchymal stem cells can survive after transplantation and apoptosis is considered an important factor that affects the curative effect (12). SDF-1 has directed chemotaxis and anti-apoptotic effects.

The present study showed that with the extension of time in the model group, the number of apoptotic cells increased accordingly. Myocardial infarction is a process of cardiomyocyte apoptosis. Cardiac remodeling after revascularization may be associated with blocking of cardiomyocytes apoptosis (13). The number of apoptotic cells in the SDF-1 intervention group was significantly less than that in the other groups at all time-points and the number of apoptotic cells on day 3 was the 

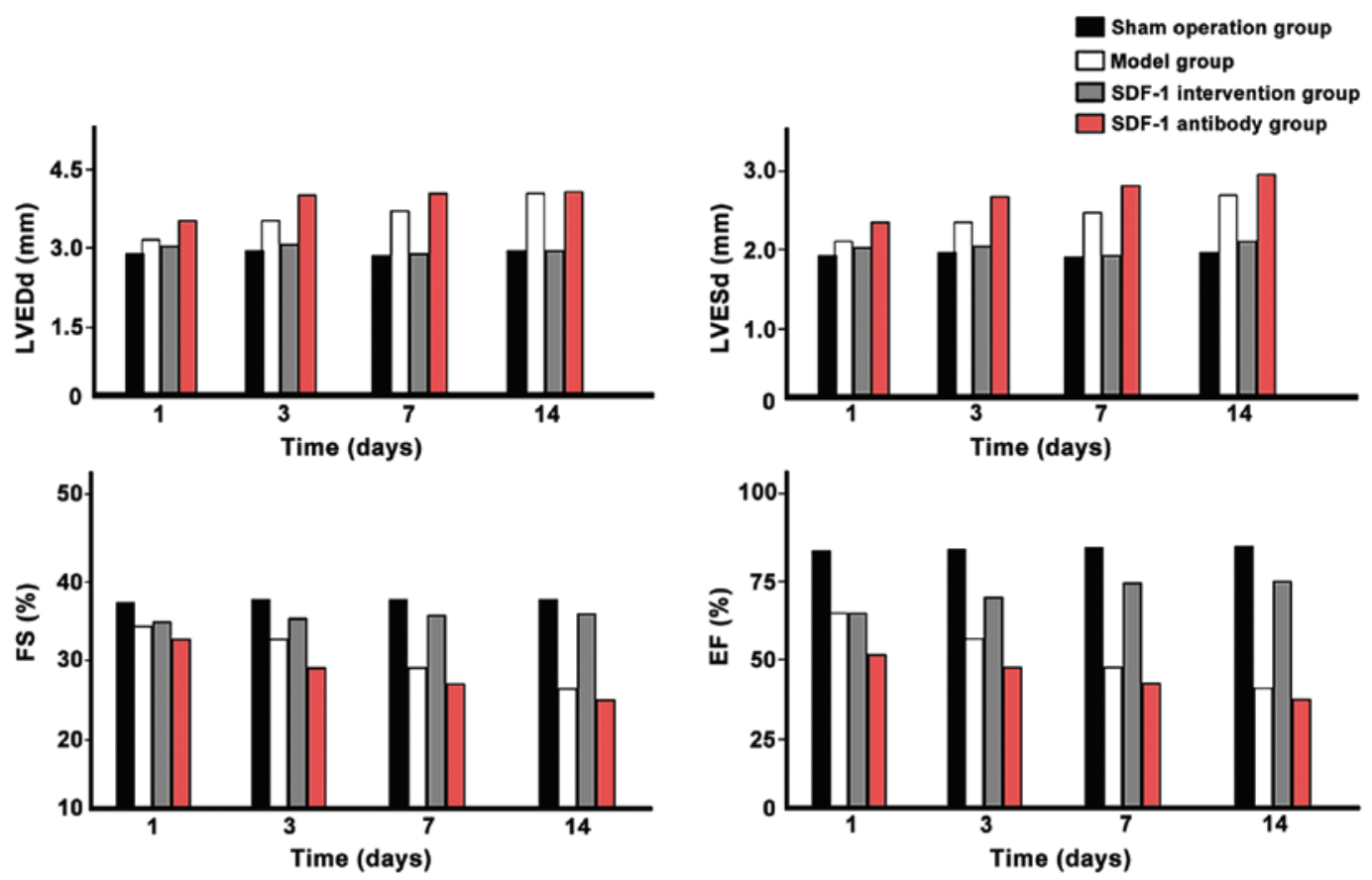

Figure 2. Cardiac ultrasound index.

Table III. Comparison of TLR-4 and NF- $\kappa \mathrm{B}$ expression levels (\%).

\begin{tabular}{|c|c|c|c|c|c|c|c|c|}
\hline \multirow[t]{2}{*}{ Groups } & \multicolumn{2}{|c|}{1 day } & \multicolumn{2}{|c|}{3 days } & \multicolumn{2}{|c|}{7 days } & \multicolumn{2}{|c|}{14 days } \\
\hline & TLR-4 & $\mathrm{NF}-\kappa \mathrm{B}$ & TLR-4 & $\mathrm{NF}-\kappa \mathrm{B}$ & TLR-4 & $\mathrm{NF}-\kappa \mathrm{B}$ & TLR-4 & $N F-\kappa B$ \\
\hline Sham operation & $1.2 \pm 0.2$ & $0.9 \pm 0.2$ & $1.3 \pm 0.3$ & $0.8 \pm 0.2$ & $1.1 \pm 0.3$ & $1.0 \pm 0.3$ & $1.0 \pm 0.3$ & $1.1 \pm 0.3$ \\
\hline Sham operation & $1.3 \pm 0.3$ & $1.0 \pm 0.3$ & $1.2 \pm 0.3$ & $0.9 \pm 0.3$ & $1.1 \pm 0.3$ & $1.0 \pm 0.3$ & $1.2 \pm 0.3$ & $0.9 \pm 0.3$ \\
\hline SDF-1 intervention & $13.2 \pm 3.2$ & $10.5 \pm 3.3$ & $21.4 \pm 5.4$ & $17.8 \pm 5.6$ & $38.7 \pm 6.6$ & $26.5 \pm 6.7$ & $40.2 \pm 8.7$ & $32.3 \pm 8.9$ \\
\hline SDF-1 antibody & $0.8 \pm 0.2$ & $0.9 \pm 0.2$ & $0.7 \pm 0.2$ & $0.8 \pm 0.2$ & $0.9 \pm 0.2$ & $0.8 \pm 0.2$ & $0.7 \pm 0.2$ & $0.7 \pm 0.2$ \\
\hline $\mathrm{F}$ & 10.532 & 12.354 & 13.624 & 14.857 & 16.359 & 18.427 & 20.312 & 23.635 \\
\hline P-value & $<0.001$ & $<0.001$ & $<0.001$ & $<0.001$ & $<0.001$ & $<0.001$ & $<0.001$ & $<0.001$ \\
\hline
\end{tabular}

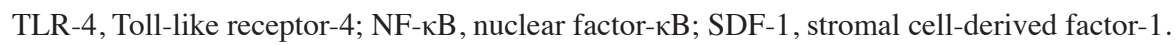

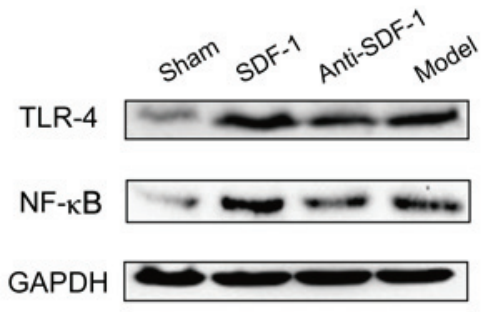

Figure 3. Western blot analysis for detection of TLR-4 and NF-кB.

greatest in number. By contrast, the number of apoptotic cells on days 7 and 14 were decreased. The number of apoptotic cells in the SDF-1 antibody group was the greatest, proving that SDF-1 obviously inhibited the process of apoptosis of cardiac muscle cells (14). The new blood vessel density in the SDF-1 intervention group was significantly higher than that in the other groups. The SDF-1 antibody group had the lowest vascular density at all time-points, suggesting that SDF-1 has the potential of cell proliferation and neovascularization. In animal models, SDF-1 and vascular endothelial growth factor (VEGF) have similar effects, which can mobilize hematopoietic stem cells and circulating EPCs (15). The mechanism of SDF-1 chemotaxis attracting circulating EPCs to ischemia or injury site may be similar to its ability of raising tissue-committed stem cells (16). Neointimal thickening was attributed to smooth muscle-like cells, and the origin of the smooth muscle-like cells is considered to be induced by the trend of SDF-1 in endothelial cells selectively up-regulating in recruiting $\mathrm{CXCR}^{+}$stem cells (17).

The LVEDd and LVESd values of each time-point in the SDF-1 intervention group were smaller than those of the model group, but larger than those of the sham operation group, decrease with time. Those in the SDF-1 antibody group at each time-point were the largest and increased with time. The 
FS and EF values of each time-point in the the SDF-1 intervention group were higher than those in the model group, but less than those in the sham operation group, increasing with time. Those in the SDF-1 antibody group at each time-point were the least and decreased with the time. It was suggested that SDF-1 can further improve the cardiac function, which may be related to its ability to inhibit myocardial apoptosis, and increase angiogenesis and chemotaxis of endogenous or exogenous pluripotent stem cell differentiation. In some studies, the autologous bone marrow stem cell of AMI patients was mobilized by subcutaneous injection of SDF-1 (18). Subsequently, peripheral blood stem cell suspension was separated. The collected stem cell suspension was injected to the infarct-related artery by over-the-wire balloon catheter in the center cavity. After 6 months, in comparison to the conventional treatment group (drugs and interventional therapy), the end systolic volume (ESV) of heart decreased significantly $(\mathrm{p}=0.01)$. The left ventricular EF increased significantly $(\mathrm{p}<0.001)$, while the left ventricular segmental wall motion score index decreased significantly $(\mathrm{p}<0.001)$, whereas the end diastolic volume (EDV) had no significant change $(p=0.07)$, indicating that SDF-1 improved the percutaneous transluminal coronary artery transplantation of autologous peripheral blood stem cells effect in the treatment of AMI for reducing myocardial infarction area, reducing left ventricular remodeling and improving cardiac function (18).

TLR-4 and NF- $\kappa$ B protein expression levels of the SDF-1 treatment group were significantly higher than those of other groups and increased with time. The difference was not statistically significant compared with those of the other groups at each time-point, suggesting that SDF-1 be involved in biological activity through the TLR-4/NF- $\kappa$ B signaling pathway (19). The TLR-4/NF- $\kappa$ B signaling pathway plays an important role in the inflammatory response mediated by inflammatory mediators such as neutrophils, inflammatory cytokines including IL-2, IL-10, $\gamma$-IFN and TNF- $\alpha$, and inflammatory mediators, which are involved in cell injury and repair. Previous findings have shown that the SDF-1/CXCR4 axis played a role through the phosphoinositide 3-kinase/protein kinase $\mathrm{C} / \mathrm{NF}-\kappa \mathrm{B}$ and p42/44 mitogen-activated protein kinase pathways $(20,21)$.

In conclusion, SDF-1 is capable of reducing the apoptosis of myocardial cells in AMI, promoting blood vessel regeneration and improving cardiac function, which may be associated with activation of the TLR-4/NF- $\kappa \mathrm{B}$ signaling pathway.

\section{References}

1. Assmus B, Leistner DM, Schächinger V, Erbs S, Elsässer A Haberbosch W, Hambrecht R, Sedding D, Yu J, Corti R, et al; REPAIR-AMI Study Group: Long-term clinical outcome after intracoronary application of bone marrow-derived mononuclear cells for acute myocardial infarction: migratory capacity of administered cells determines event-free survival. Eur Heart J 35: 1275-1283, 2014.

2. Ripa RS and Kastrup J: Stem cells: REGENT trial-the end of cell therapy for MI? Nat Rev Cardiol 6: 567-568, 2009.

3. Meyer GP, Wollert KC, Lotz J, Pirr J, Rager U, Lippolt P, Hahn A, Fichtner S, Schaefer A, Arseniev L, Ganser A and Drexler H: Intracoronary bone marrow cell transfer after myocardial infarction: 5-Year follow-up from the randomized-controlled BOOST trial. Eur Heart J 30: 2978-2984, 2009.

4. Zgraggen S, Huggenberger R, Kerl K and Detmar M: An important role of the SDF-1/CXCR4 axis in chronic skin inflammation. PLoS One 9: e93665, 2014.
5. Youssef A and Han VK: Low oxygen tension modulates the insulin-like growth factor-1 or -2 signaling via both insulin-like growth factor-1 receptor and insulin receptor to maintain stem cell identity in placental mesenchymal stem cells. Endocrinology 157: 1163-1174, 2016.

6. Dalonneau F, Liu XQ, Sadir R, Almodovar J, Mertani HC, Bruckert F, Albiges-Rizo C, Weidenhaupt M, Lortat-Jacob H and Picart C: The effect of delivering the chemokine SDF-1a in a matrix-bound manner on myogenesis. Biomaterials 35: 4525-4535, 2014.

7. Mierzejewska K, Klyachkin YM, Ratajczak J, Abdel-Latif A, Kucia M and Ratajczak MZ: Sphingosine-1-phosphate-mediated mobilization of hematopoietic stem/progenitor cells during intravascular hemolysis requires attenuation of SDF-1-CXCR4 retention signaling in bone marrow. BioMed Res Int 2013: 814549, 2013.

8. Weidner N: Intratumor microvessel density as a prognostic factor in cancer. Am J Pathol 147: 9-19, 1995.

9. Rudski LG, Lai WW, Afilalo J, Hua L, Handschumacher MD, Chandrasekaran K, Solomon SD, Louie EK and Schiller NB: Guidelines for the echocardiographic assessment of the right heart in adults: A report from the American Society of Echocardiography endorsed by the European Association of Echocardiography, a registered branch of the European Society of Cardiology, and the Canadian Society of Echocardiography. J Am Soc Echocardiogr 23: 685-713, 786-788, 2010.

10. Gong J, Meng HB, Hua J, Song ZS, He ZG, Zhou B and Qian MP: The SDF-1/CXCR4 axis regulates migration of transplanted bone marrow mesenchymal stem cells towards the pancreas in rats with acute pancreatitis. Mol Med Rep 9: 1575-1582, 2014.

11. Roy LD, Sahraei M, Schettini JL, Gruber HE, Besmer DM and Mukherjee P: Systemic neutralization of IL-17A significantly reduces breast cancer associated metastasis in arthritic mice by reducing CXCL12/SDF-1 expression in the metastatic niches. BMC Cancer 14: 225-227, 2014.

12. Belle JI, Petrov JC, Langlais D, Robert F, Cencic R, Shen S, Pelletier J, Gros P and Nijnik A: Repression of p53-target gene Bbc3/PUMA by MYSM1 is essential for the survival of hematopoietic multipotent progenitors and contributes to stem cell maintenance. Cell Death Differ 23: 759-775, 2016.

13. Chang J, Zhang G, Zhang L, Hou YP, Liu XL and Zhang L: High admission glucose levels increase Fas apoptosis and mortality in patients with acute ST-elevation myocardial infarction: a prospective cohort study. Cardiovasc Diabetol 12: 171, 2013.

14. Fan Y, Yang F, Cao X, Chen C, Zhang X, Zhang X, Lin W, Wang $X$ and Liang $C$ : Gab1 regulates SDF-1-induced progression via inhibition of apoptosis pathway induced by PI3K/AKT/Bcl-2/BAX pathway in human chondrosarcoma. Tumour Biol 16: 12-14, 2015.

15. Moore MA, Hattori K, Heissig B, Shieh JH, Dias S, Crystal RG and Rafii S: Mobilization of endothelial and hematopoietic stem and progenitor cells by adenovector-mediated elevation of serum levels of SDF-1, VEGF, and angiopoietin-1. Ann N Y Acad Sci 938: 36-45, discussion 45-47, 2001.

16. Urbich C and Dimmeler S: Endothelial progenitor cells: characterization and role in vascular biology. Circ Res 95: 343-353, 2004.

17. Ratajczak MZ, Majka M, Kucia M, Drukala J, Pietrzkowski Z, Peiper S and Janowska-Wieczorek A: Expression of functional CXCR4 by muscle satellite cells and secretion of SDF-1 by muscle-derived fibroblasts is associated with the presence of both muscle progenitors in bone marrow and hematopoietic stem/progenitor cells in muscles. Stem Cells 21: 363-371, 2003.

18. Gao C and Li Y; GaoC and Li Y: SDF-1 plays a key role in the repairing and remodeling process on rat allo-orthotopic abdominal aorta grafts. Transplant Proc 39: 268-272, 2007.

19. Xing Q, de Vos P, Faas MM, Ye Q and Ren Y: LPS promotes pre-osteoclast activity by up-regulating CXCR4 via TLR-4. J Dent Res 90: 157-162, 2011.

20. Džumhur A, Zibar L, Wagner J, Simundić T, Dembić Z and Barbić J: Association studies of gene polymorphisms in toll-like receptors 2 and 4 in Croatian patients with acute myocardial infarction. Scand J Immunol 75: 517-523, 2012.

21. Roland J, Murphy BJ, Ahr B, Robert-Hebmann V, Delauzun V, Nye KE, Devaux C and Biard-Piechaczyk M: Role of the intracellular domains of CXCR4 in SDF-1-mediated signaling. Blood 101: 399-406, 2003. 